\title{
Kawasaki Disease: The Debate Continues Till Date
}

\section{Rajinder M Joshi* \\ Microbiology Unit Radiology, Yiaco Medical Co. Al Adan Hospital, Kuwait}

Tomisaku Kawasaki, a pediatrician saw a 4 years old child in 1961 with features of an unknown disease later known as Kawasaki disease (KD) in his honor. He presented his findings in a clinical conference at the Red Cross Hospital in Hiroo, Tokyo. A year later he came across yet another child with similar presentation. In the following years he happened to see about 50 more such patients. He published his findings in 1967 in a local medical journal in Japanese language [1]. The English version of the disease was published only in 1974 [2]. Despite the disease having been reported from almost all parts of the world ever since 1970s and the extensive advancement in the medical field, the debate on the etiology, clinical presentation, epidemiology and treatment of $\mathrm{KD}$ continues till date. $\mathrm{KD}$ is an acute febrile illness with multi organ vasculitis primarily affecting infants and young children under 5 years of age. It is also known as Mucocutaneous Lymph Node Syndrome (MLNS), infantile vasculitis, Kawasaki syndrome and immune vasculitis. Usually small to medium sized blood vessels are affected in KD. The male children are affected one and a half times more than female ones. The disease also sometimes affects children over 5 years of age and is rarely encountered in adults. Most of the patients are of Japanese and pacific islanders origin and is reported more often from developed world including USA in particular. The disease has now been increasingly reported from all over the world including the developing countries [3-8]. It may have some seasonal variations. Genetic constitution may be responsible for increased susceptibility in some patients. Clinical features and epidemiological findings suggest that KD may be caused by an infectious agent. Various agents like rug shampoo, bacteria, viruses, parasites, fungi and their super antigens have been incriminated in the pathogenesis of the disease. Recently a number of reports associate the streptococci with the KD [9-12]. A more recent report concludes that our knowledge of the infectious agent (s) involved and the genetic characteristics of susceptible children remain only partial [13].

The KD which is now regarded as a leading cause of acquired heart disease in the developed world starts abruptly with high grade fever lasting 5 days or more with at least four of the following other features i.e. bilateral non purulent conjunctivitis; mucosal inflammation including sore/cracked lips, inflammed pharynx or strawberry tongue; erythema of the palms and soles, desquamation of fingers and toes and peripheral non-pitting oedema; polymorphous rash; or cervical lymphadenopathy. The American and the Japanese criteria of KD definition may differ slightly. However, about $10 \%$ of children still do not meet the strict criteria. These cases may fall into sub acute, incomplete or atypical KD. The disease has a predilection for cardiac tissues in particular the coronary arteries. Although most of the patients recover from acute symptoms even if untreated, the risk of the cardiac complications may be high. Without treatment, 20 to $25 \%$ of patients develop cardiac complications [14]. The latter include coronary aneurysms, ischemic heart disease, myocarditis, coronary thrombus, myocardial infarction and sudden heart failure. It is also debatable if the KD predisposes the host to atherosclerosis. About $1 \%$ patients especially with giant coronary aneurysms may die. However, long term mortality may be much higher. It is relevant to mention here that many of the coronary aneurysms detected in adults may be possibly attributed to KD acquired in their childhood. Depending upon various phases of the disease some patients may even present with aseptic meningitis, hyperemic tympanic membrane, uveitis, diarrhoea, abdominal pain, pneumonitis, facial nerve palsy, seizures, ataxia, cerebral infarctions, gallbladder hydrops, arthritis, erythema and induration at the recent site of BCG vaccination, testicular swelling and peripheral gangrene. The disease should be carefully differentiated from scarlet fever, toxic shock syndrome, measles, glandular fever, Stevens -Johnson syndrome and viral meningitis.

Radiological investigations may be useful for cardiac assessment. Chest radiography, CT and MRI are not routinely done. Echocardiography especially 2-dimensional may have 100\% sensitivity and about $90 \%$ specificity in detecting coronary aneurysms. While CT may be more sensitive in detecting coronary calcification, MRI may be useful for long term cardiac follow up.

Patients with KD should be hospitalized as early as possible and standard treatment may be instituted within 24 hours or at least within 10 days of onset of fever which will help minimize complications. Intravenous gammaglobulin (IVIG) $2 \mathrm{gm} / \mathrm{kg}$ as a single dose is the most effective remedy. This may be combined with high dose aspirin, though the role of aspirin has been debatable. Some clinicians do not advocate aspirin therapy lest the children should develop Reye's syndrome. If the fever does not respond to the first dose of IVIG within 24 hours, a second dose may be administered. The third dose of IVIG is rarely recommended. The cases refractory to IVIG may require steroids, infliximab, low dose methotrexate, cyclophosphamide or even plasma exchange. Etanercept therapy is also currently under consideration for such cases.

It appears that though a few pathogens may be capable of triggering immune vasculitis seen in $\mathrm{KD}$, the overall pathogenesis of the disease is largely determined by the genetic constitution/susceptibility of the host. It is probably more pertinent and logical here to state that it is the soil (host) that counts more than the seed (infecting agent) in the genesis of the disease. Given the subtle and variable clinical presentation of the disease and subsequent serious cardiac complications, any parsimonious attitude especially in requesting for an echocardiogram may not be justifiable. While a pragmatic approach in near future may elucidate more facts about the etio pathogenesis of this disease, the general awareness particularly in the developing world may lead to effectively reduce both the immediate and late morbidity and mortality which may ensue months, years and even decades after the initial illness.

*Corresponding author: Rajinder M Joshi, Microbiology Unit Radiology, Nuclear Medicine and Laboratory Center, Yiaco Medical Co. Al Adan Hospital, Kuwait, E-mail: drrmjoshi2002@yahoo.com

Received May 28, 2013; Accepted June 03, 2013; Published June 06, 2013

Citation: Joshi RM (2013) Kawasaki Disease: The Debate Continues Till Date. Clin Microbial 2: e112. doi:10.4172/2327-5073.1000e112

Copyright: @ 2013 Joshi RM. This is an open-access article distributed under the terms of the Creative Commons Attribution License, which permits unrestricted use, distribution, and reproduction in any medium, provided the original author and source are credited. 


\section{References}

1. Kawasaki T (1967) [Acute febrile mucocutaneous syndrome with lymphoid involvement with specific desquamation of the fingers and toes in children]. Arerugi 16: 178-222.

2. Kawasaki T, Kosaki F, Okawa S, Shigematsu I, Yanagawa H (1974) A new infantile acute febrile mucocutaneous lymph node syndrome (MLNS) prevailing in Japan. Pediatrics 54: 271-276.

3. Khubchandani R, D'Souza S (2001) Kawasaki Disease in India. Pediatric Rheumatol Online; 2

4. Kushner HI, Macnee RP, Burns JC (2009) Kawasaki disease in India: increasing awareness or increased incidence? Perspect Biol Med 52: 17-29.

5. Nakamura Y, Yashiro M, Uehara R, Sadakane A, Chihara I, et al. (2010) Epidemiologic features of Kawasaki disease in Japan: results of the 2007-2008 nationwide survey. J Epidemiol 20: 302-307.

6. Du ZD, Zhao D, Du J, Zhang YL, Lin Y, et al. (2007) Epidemiologic study on Kawasaki disease in Beijing from 2000 through 2004. Pediatr Infect Dis J 26 449-451.

7. Bar-Meir M, Haklai Z, Dor M (2011) Kawasaki disease in Israel. Pediatr Infect Dis J 30: $589-592$.
8. Singh S, Aulakh R, Bhalla AK, Suri D, Manojkumar R, et al. (2011) Is Kawasak disease incidence rising in Chandigarh, North India? Arch Dis Child 96: 137 140.

9. Akiyama T, Osawa N, Shimanuki K, Yashiro K, Oyake T (1991) Possible role of Streptococcus pyogenes in mucocutaneous lymph node syndrome. XII. Variable responses of platelets in MCLS seem to be explainable by streptococcal pyrogenic exotoxin. Acta Paediatr Jpn 33: 20-26.

10. Anderson DG, Warner G, Barlow E (1995) Kawasaki disease associated with streptococcal infection within a family. J Paediatr Child Health 31: 355 357

11. Bourusly M, Jawad N (2005) Kawasaki Disease in a Kuwaiti family. Kuwait Med J 37: 110-112.

12. Joshi RM, Al Azemi M, Al Ismail AA (2009) Streptococcus Group A Septicemia with Kawasaki Disease: A Case Report. The New Egypt J Med 41(3): 283-285

13. Principi N, Rigante D, Esposito S (2013) The role of infection in Kawasak syndrome. J Infect .

14. Taubert KA, Shulman ST (1999) Kawasaki disease. Am Fam Physician 59 3093-3102, 3107-8. 